\title{
SIR-ZEE: plataforma tecnológica para el desarrollo local
}

\author{
SIR-ZEE: technology platform \\ for local development
}

Marlen Treviño Villalobos'

Marcela Chaves Álvarez²

Fecha de recepción: 20 de enero del 2012 Fecha de aprobación: 19 de marzo del 2012

Este artículo cuenta con el aval de la

Vicerrectoría de Investigación y Extensión del Tecnológico de Costa Rica
Treviño, M; Chaves, M. SIR-ZEE: plataforma tecnológica para el desarrollo local. Tecnología en Marcha. Vol. 25, № 5. Pág 93-102.
Ingeniera en computación. Departamento de Computación, Instituto Tecnológico de Costa Rica, sede San Carlos. Teléfono: 240 I-3|48. Correo electrónico: mtrevino@itcr.ac.cr

2 Ingeniera civil. Departamento de Computación, Instituto Tecnológico de Costa Rica, sede San Carlos. Teléfono: 240I-3|48. Correo electrónico: mchaves@itcr.ac.cr 


\section{Resumen}

El Sistema de Información Regional de la Zona Económica Especial (SIR-ZEE) nació en Costa Rica aproximadamente en 1998, cuando se concibió la idea de crear un sistema informático para recopilar, gestionar y ofrecer temáticas generadas en forma digital por diferentes entidades públicas y privadas. En el 2008, con los fondos del Programa de Regionalización Interuniversitaria del Consejo Nacional de Rectores, se aprobó la iniciativa SIR-ZEE, Plataforma Tecnológica para el Desarrollo Local, que buscó fortalecer las capacidades productivas y las capacidades de gobernabilidad en la Región Huetar Norte mediante el acceso a la información oportuna y relevante para el desarrollo económico local.

Este artículo tiene como objetivo mostrar no solo la relevancia de un sistema de información en la planificación del desarrollo local, sino también que, en la actualidad, el SIR-ZEE se está convirtiendo en una ventaja competitiva para la Región Huetar Norte, ya que facilita el proceso de toma decisiones. Los principales resultados obtenidos al final de la iniciativa fueron una propuesta metodológica para la elaboración de planes estratégicos de desarrollo local en la Región Huetar Norte, la sensibilización de los actores locales sobre la participación ciudadana en las diferentes fases de la planificación, un diagnóstico territorial con el valor agregado de contar con la información georreferenciada y disponible en un sitio web, y avances en la elaboración del Plan estratégico de desarrollo local.

\section{Palabras clave}

Sistema de información, desarrollo local, planificación y Región Huetar Norte.

\section{Abstract}

The Regional Information System of the Special Economic Zone (SIR-ZEE) was born about the year 1998 as a computer system to collect, manage and offer thematic generated in digital format by different public and private entities. In 2008, with the funds of Interuniversity Regionalization Program the National Council of Rectors approved the initiative SIRZEE: Platform Technology for Local Development which sought to strengthen productive capacities and capabilities of governance in the North Huetar Region through access to timely and relevant to the local economic development.

This article aims to present the relevance of an information system in local development planning and currently the SIR-ZEE is becoming a competitive advantage for the North Huetar Region facilitating decision-making process. The main results obtained at the end of the initiative was a proposal to the processing of strategic plans for local development in the North Huetar Region, awareness of local stakeholders on the participation in the various stages of planning, a territorial diagnosis with the added value of having the information geo-referenced and available on the web site and progress in the Strategic Plan for Local Development.

\section{Key words}

Information system, local development, planning and North Huetar Region. 


\section{Introducción}

El Sistema de Información Regional de la Zona Económica Especial (SIR-ZEE) nació aproximadamente en 1998, cuando se dieron los primeros pasos para establecer las bases de un sistema de información para la Región Huetar Norte de Costa Rica (Wild y López, 2008). Posteriormente, entre abril del 2004 y diciembre del 2006 se efectuó el proceso de implementación y consolidación de este.

EI SIR-ZEE es un sistema informático para recopilar, gestionar y ofrecer temáticas generadas en forma digital por diferentes entidades públicas y privadas. El acceso a dichas temáticas favorece su utilización tanto en los procesos productivos de las pymes, como en el proceso de toma de decisiones de los gobiernos locales de la Región Huetar Norte.

Este sistema se desarrolló gracias al concurso de cinco instituciones: el Programa de las Naciones Unidas para el Desarrollo (PNUD), el Ministerio de Relaciones Exteriores y Culto (RREE), la Embajada de Japón, la Agencia para el Desarrollo de la Región Huetar Norte (referida como Zona Económica Especial (ZEE)) y el Instituto Tecnológico de Costa Rica (TEC).

En el 2008, se plantearon tres proyectos que finalizaron en diciembre del 20ll, financiados con los fondos del Programa de Regionalización Interuniversitaria del Consejo Nacional de Rectores, el cual busca la articulación de la acción académica de sus miembros en favor de las regiones periféricas del país. Con este propósito, utiliza la ejecución de Iniciativas Interuniversitarias de Desarrollo Regional
(IIDR) dirigidas a incidir en el desarrollo. Dos de estas iniciativas tratan de transferir la experiencia a otras regiones del país, mientras que la tercera propuesta busca impactar en el área de influencia del SIR-ZEE (ver cuadro I), y se denominó "SIR-ZEE: Plataforma Tecnológica para el Desarrollo Local".

En la propuesta inicial de la IIDR Plataforma Tecnológica para el desarrollo local, el área de trabajo fue la Región Huetar Norte de Costa Rica. Sin embargo, por la complejidad de los objetivos planteados, así como por la limitación de recursos, se decidió acotar el territorio. De este modo, para el 2010 se seleccionó el cantón de Guatuso para realizar un plan piloto y en el 20 II se continuó el trabajo en el cantón de Los Chiles.

Es importante mencionar que en esta IIDR participó un equipo de trabajo multidisciplinario conformado por tres funcionarios de la Carrera de Computación de San Carlos del Instituto Tecnológico de Costa Rica, el Instituto de Investigación y Servicios Forestales de la Universidad Nacional, el Departamento de TIC de Sarapiquí de la Universidad Nacional, el Centro Internacional de Política Económica Para el Desarrollo Sostenible (CINPE) de la Universidad Nacional, el Observatorio del Desarrollo de la Universidad de Costa Rica, el Centro de Investigaciones Agronómicas de la Universidad de Costa Rica, el Programa de Educación Técnica - Guápiles de la Universidad de Costa Rica, y la Carrera de Ingeniería en Informática de la Universidad Nacional Estatal a Distancia. Además, es de suma relevancia describir por cada línea de trabajo los principales beneficiarios de la IIDR:

Cuadro I. Proyectos en Regionalización Interuniversitaria

\begin{tabular}{|l|l|}
\hline \multicolumn{1}{|c|}{ Proyecto } & \multicolumn{1}{c|}{ Descripción } \\
\hline $\begin{array}{l}\text { Plataforma tecnológica para } \\
\text { el desarrollo económico } \\
\text { local }\end{array}$ & $\begin{array}{l}\text { Se trata de una iniciativa, en el marco del Sistema SIR-ZEE, que busca tres objetivos } \\
\text { específicos:a.fortalecer el Sistema SIR-ZEE en función de la competitividad empresarial de } \\
\text { la Región Huetar Norte; b. fortalecer y desarrollar centros de acceso a la información en } \\
\text { diferentes comunidades de la Región Huetar Norte; y c. generar y transferir capacidades } \\
\text { locales para el mejor aprovechamiento de la información y tecnología disponible en el } \\
\text { SIR-ZEE. Las acciones del proyecto conllevan a desarrollar cuatro líneas de trabajo: a. } \\
\text { Subsistema de planeación del desarrollo local; b. Subsistema de fertilidad de suelos; c. Red } \\
\text { local de infocentros; y d. Técnicos universitarios. }\end{array}$ \\
\hline SIR-PC y SIR-SUR & $\begin{array}{l}\text { Las IIDR, denominadas Sistema de Información Regional del Pacífico Sur (SIR-SUR) y } \\
\text { Sistema de Información Regional del Pacífico Central (SIR-PC), tienen como objetivo } \\
\text { general "impulsar las capacidades productivas en la Región Pacífico Sur y Pacífico Central } \\
\text { a través del acceso a la información oportuna y relevante para el desarrollo económico } \\
\text { local, la que estará concentrada en un Sistema de Información Regional". }\end{array}$ \\
\hline
\end{tabular}

Fuente: Elaboración propia. 
- Planeación del desarrollo

- Quince funcionarios de las municipalidades de Guatuso, Upala y Los Chiles, quienes reciben una capacitación sobre planificación estratégica municipal.

- Pobladores del cantón de Los Chiles y de Guatuso.

- Veinte funcionarios del Ministerio de Salud.

- ASADAS del cantón de Guatuso.

- Docentes y estudiantes de informática en los siguientes colegios de la Dirección Regional de Educación de San Carlos: CTP San Carlos (COTAI) Ciudad Quesada, CTP Fortuna, Liceo Experimental Bilingüe de Los Ángeles de La Fortuna y Liceo de Chachagua.

- Fertilidad de suelos

- Ciento veinte productores de los cantones de San Carlos, Guatuso, Upala y Los Chiles recibieron, de forma gratuita, su análisis de muestras de suelos.

- Veintidós productores del cantón de Guatuso se capacitaron en recolección y análisis de muestras de suelos y biocontroladores.

- Programa de técnicos

- Veinte empresarios del cantón de San Carlos de comunidades como Bajo Rodríguez, Los Ángeles, La Fortuna, Florencia, La Tigra, Chachagua y Santa Rosa de Pocosol.

- Veinte empresarios del cantón de Sarapiquí.

- Veintitrés empresarios del cantón de Guápiles.

Asimismo, para el 2012 fueron aprobados tres nuevos proyectos en el Programa de Regionalización Interuniversitaria, entre los que se destaca el apoyo en la gestión del catastro en las municipalidades de Guatuso, Upala y Los Chiles mediante la transferencia de tecnología. En este proyecto trabajan en conjunto la Escuela de Topografía de la Universidad Nacional, el Instituto de Formación Municipal de la Universidad Estatal a Distancia y la Carrera de Computación del Instituto Tecnológico de Costa Rica.

Como se aprecia, el SIR-ZEE ha evolucionado en la gama de servicios que ofrece, en su organización y, sobre todo, en la vinculación con los actores del desarrollo local de la Región Huetar Norte del país.
Por ello, en este artículo se pretende presentar la forma en que un Sistema de Información Regional puede ser una herramienta para la planificación del desarrollo local.

El resto del artículo se distribuye en cuatro secciones adicionales. En la segunda parte se presenta la definición de los principales conceptos relacionados con la planificación del desarrollo local. En la tercera sección se muestra el caso de estudio de este trabajo, y en la cuarta se presentan los resultados del estudio. Finalmente, las conclusiones y trabajos futuros se incluyen en la quinta sección, que cierra el artículo.

\section{Planificación del desarrollo local}

En este apartado se presenta una breve conceptualización de tres ejes temáticos: desarrollo local, planificación estratégica y sistemas de información; con el objetivo de analizar el papel de los sistemas de información como elemento clave de la planificación estratégica del desarrollo local.

\section{Desarrollo local}

El desarrollo local constituye, en la actualidad, un tema de amplia reflexión, pues el objetivo primordial de la mayoría de los países es elevar el bienestar y la capacidad económica de la población. Para entender este término, primero es necesario conocer el concepto "desarrollo endógeno" y cuáles son las razones que nos llevan a hablar y practicar este tema.

Se habla de desarrollo endógeno, en primer lugar, porque vivimos en un nuevo contexto conocido como globalización; en segundo lugar, porque la globalización genera impactos de diversa índole en los territorios; porque existen nuevas dimensiones territoriales a tomar en cuenta: lo global, lo nacional y lo local; y por último, porque existen nuevas maneras de abordar el desarrollo.

La Teoría del Desarrollo Endógeno se refiere al conjunto de ideas y supuestos que articulan y organizan los distintos conceptos asociados al desarrollo pensando desde los territorios (Fundación Demuca y CEDeT, 2009). Este enfoque indica que el desarrollo no se preocupa exclusivamente por las condiciones de reproducción de capital, sino también por la calidad de vida de las personas en el territorio. Es desde esta perspectiva que el enfoque 
del desarrollo endógeno resulta aplicable en los procesos de desarrollo local, regional o territorial.

Después de analizar de dónde proviene el término "desarrollo local", es necesario tener una definición formal del concepto. Por ello, se hace referencia a la definición de Sergio Buarque (1999), la cual indica que el desarrollo local es "un proceso endógeno registrado en pequeñas unidades territoriales y asentamientos humanos capaz de promover el dinamismo económico y la mejoría en la calidad de vida de la población".

A pesar de construir un movimiento de fuerte contenido interno, el desarrollo local está inserto en una realidad más amplia y compleja como la que integra, y de la cual recibe influencias y presiones. "El concepto genérico de desarrollo local puede ser aplicado para diferentes cortes territoriales o aglomerados humanos de pequeña escala, desde una comunidad hasta el municipio o micro regiones homogéneas de porte reducido. El desarrollo municipal es por tanto un caso particular de desarrollo local con una amplitud espacial delimitada por el corte político" (Buarque, 1999).

Una vez presentado el concepto de desarrollo local, es importante conocer quiénes son los protagonistas del desarrollo. Los territorios están constituidos por una sociedad local que es un sistema de relaciones sociales, en donde se encuentran actores locales y extra-locales. Los actores locales son sujetos sociales cuyos comportamientos inciden en la comunidad en la que están insertos pero que, a la vez, se encuentran condicionados por las particularidades de su entorno. La actuación coordinada de los diferentes actores locales y la relación de estos con otros de nivel regional y nacional hace posible el desarrollo de una serie de condiciones necesarias para el desarrollo local, en el marco de un enfoque sistémico e integral del desarrollo.

\section{Planificación estratégica}

La aparición y auge de la planificación estratégica en la gestión local se debe a la necesidad de las ciudades y sus gobiernos de dar respuesta a una serie de retos y nuevas situaciones que convierten el territorio en un elemento básico de la competitividad.

Antes de continuar, es necesario conocer qué significa el término Planificación Estratégica. El Diccionario de la Real Academia Española (Real Academia Española,
200 I) define planear como "trazar o formar el plan de una obra", y estrategia como "arte para dirigir un asunto"; por lo cual se podría decir que mediante la planificación estratégica se pretende dar orden y dirección al quehacer y desarrollo de un sistema, sea este una empresa, un ente territorial o localidad, o cualquier tipo de organización (Ríos, 2007).

Ahora, surge la pregunta ipor qué planear estratégicamente el desarrollo local?, a lo que Durys Ríos (2007) responde que si el desarrollo local se concibe como un proceso de crecimiento y cambio estructural, en el que se busca explotar las potencialidades de una localidad e incrementar sus niveles de eficiencia y competitividad de manera que se logre una mejor calidad de vida para los actores, el proceso a seguir debe ser consciente y ordenado, es decir un proceso planeado estratégicamente. También indica que la forma de planear el desarrollo por parte de los gobiernos no es la más adecuada, ya que se basa en tendencias pasadas y los planes se encuentran enmarcados en una lógica cortoplacista. Asimismo, las localidades se encuentran inmersas en un entorno de globalización y descentralización.

La planificación estratégica constituye una técnica que está directamente ligada al Desarrollo Local, pues representa un proceso de prevención a largo plazo, que podría ayudar a las localidades en su reflexión acerca del futuro, y en la definición y ejecución de los planes de desarrollo.

\section{Sistemas de información territorial}

Un sistema de información es un instrumento de gestión conformado por un conjunto organizado de datos con una finalidad u objetivo preestablecido según los usuarios o destinatarios de dicha información. La materia prima es siempre datos, elementos aislados que, luego de ser almacenados, procesados y distribuidos, se convierten en información útil (Cavuota, 2009).

Pero, en este caso, se debe profundizar en la gama de sistemas de información y, entre ellos, definir el término Sistema de Información Territorial (SIT). Cavuota menciona que un SIT es un espacio permanente de análisis, propuestas, coordinación, acción y evaluación sobre la situación económicosocial de la región, con la presencia y participación de todos los actores comprometidos con el desarrollo planificado y sostenible de ella. 
También es importante mencionar que los principales temas que debe contemplar un SIT son la información del territorio y de su entorno, considerando lo local como sistema abierto, así como comprender las relaciones e interrelaciones que se producen con el ambiente mayor. EI SIT debe contener la mejor caracterización del entorno empresarial, es decir, las infraestructuras y equipamientos básicos, el sistema educativo y de formación de los recursos humanos, la investigación y desarrollo para la innovación $(\mathrm{I}+\mathrm{D}+\mathrm{i})$, o el marco jurídico y regulatorio (Cavuota, 2009).

De igual manera, debe contener información tanto sobre los aspectos tangibles del desarrollo como sobre los intangibles, por ejemplo, la capacidad emprendedora, las iniciativas de asociatividad empresaria, las redes o tramas de relaciones de interés mutuo, los aspectos culturales dinámicos, y los procesos de innovación, bien sean por creación o por incorporación de avances tecnológicos. Asimismo, debe destacarlos y comunicarlos para provocar la imitación y, de esta forma, potenciar las capacidades del territorio.

\section{Sistemas de información territorial, planificación estratégica y desarrollo local}

En la noción de desarrollo local se encuentra implícito un reconocimiento de la localidad como un espacio geográfico con características específicas, que se traducen en una problemática socioeconómica única y que requiere de una solución originada "desde abajo"; por lo que los tradicionales planes de desarrollo elaborados desde el poder central, y de carácter agregado y sectorial, no logran dar solución a las problemáticas propias de cada localidad.

Para lograr un cambio estructural, basado en estos tres conceptos, se deben aprovechar los recursos potenciales de cada localidad y el proceso debe ser concertado entre los diversos agentes o actores del desarrollo, pues estos cumplen un papel fundamental, al ser los gestores de su propio bienestar y quienes construyen su futuro. Por tal motivo, resulta necesario planificar el proceso del desarrollo local; en este sentido, la planificación estratégica constituye una opción acorde a estas necesidades (Ríos, 2007).

No obstante, para poder planificar es necesario conocer, y para ello es indispensable la información, la que luego se complementa con las percepciones que los actores manifiestan en los procesos participativos. Es en este momento donde se integra el concepto de Sistema de Información Territorial, que, si bien no es imprescindible para la planificación del desarrollo, puede convertirse en una ventaja competitiva para la región, lo que facilitaría el proceso de toma decisiones.

\section{Caso de estudio}

El área de trabajo de la iniciativa Plataforma tecnológica para el desarrollo local comprendió los cantones de San Carlos, Guatuso, Los Chiles y Upala, de la Provincia de Alajuela, y el cantón de Sarapiquí de la provincia de Heredia; además de los distritos de San Isidro de Peñas Blancas, Río Cuarto de Grecia y Sarapiquí de la provincia de Alajuela. Juntos conforman la Región Huetar Norte, que tiene una extensión aproximada de 9603,4 km², lo que equivale a un $18,4 \%$ del territorio nacional.

\section{Objetivo del estudio}

El objetivo de este trabajo es mostrar la relevancia de un sistema de información en la planificación del desarrollo local.

\section{Resultados y discusión}

En este apartado se presentarán los principales resultados obtenidos en la línea de trabajo Planeación del desarrollo local, así como su función en dicha planeación.

\section{Metodología}

El primer resultado obtenido en este proceso fue la propuesta metodológica para la elaboración de planes estratégicos de desarrollo local en la Región Huetar Norte. En esta propuesta se encuentran los elementos básicos que no pueden estar ausentes en el proceso de diseño de un Plan estratégico para el desarrollo local. Pero hay que considerar que las distintas condiciones que caracterizan a los municipios llevan a pensar que no hay metodología única que sirva para todos, por lo que se busca dar orientaciones generales, que necesariamente habrá que adaptar a cada caso.

Esta propuesta metodológica está basada en el libro Guía de herramientas municipales para la promoción del desarrollo económico local de la Fundación Demuca (Fundación Demuca, 2009), y el estudio 
de caso desarrollado en la Comunidad 14 de julio (Águila, Moya y Becerro, 2009) en Cuba.

La metodología de planificación estratégica propuesta para desarrollar el plan estratégico territorial en la región tiene implícitos tres procesos que subyacen en las etapas o fases del proceso de planeamiento; estos son:

a. El proceso técnico: es una dinámica de trabajo de medición y análisis de la realidad del territorio, que incluye las tendencias, así como los factores externos e internos que condicionan el desarrollo. Asimismo, se identifican las aspiraciones, sueños de los agentes y los procesos que ellos reconocen como determinantes para explicar la realidad del espacio local en el que se está interviniendo.

b. El proceso participativo: es una dinámica de construcción de espacios de concertación y consenso sobre aspectos estratégicos para el desarrollo, que supone generar corrientes de opinión pública y comunicación local que permitan formar nuevos valores y conocimientos que afirmen la identidad cultural y el ejercicio de deberes y derechos.

c. El proceso político: es una dinámica que tiene que ver con el poder local, con el reconocimiento de que el líder natural del proceso es la autoridad elegida por voto popular y que, mientras esté en ejercicio de ese poder legítimamente alcanzado, es su competencia y responsabilidad convocar y articular a los actores y agentes del desarrollo, denominación que comprende organizaciones sociales de base vinculadas a la actividad productiva, iglesia, ONGs e instituciones públicas involucradas. El proceso político incorpora las iniciativas y procesos que dan paso a la generación de compromisos y alianzas estratégicas que posibilitan el desarrollo.

Con respecto a las fases (ver figura I), se tiene como punto de partida la sensibilización de actores del desarrollo local, con el fin de conseguir su participación activa en el proceso, así como fortalecer las capacidades locales. En la fase I se realiza un diagnóstico territorial en el que se hace un análisis detallado de la situación pasada y presente del territorio. En la fase 2 se efectúa el proceso para la elaboración del Plan, en el que se llevan a cabo una serie de talleres para identificar potencialidades, escenarios de futuro, la visión, políticas, objetivos y acciones. Por último, la fase 3 es un eje transversal a todo el proceso y consiste en desarrollar un sistema vigía para monitorear la ejecución del plan.

\section{Sensibilización de actores}

En la fase de sensibilización de actores, se conformó un equipo de gestión local en el cantón de Guatuso, en el que actualmente se encuentran trabajando ocho personas. Se realizaron tres jornadas de trabajo denominadas:

d. Sensibilización para una participación activa en la elaboración del Plan estratégico de desarrollo local del cantón de Guatuso.

e. Información del cantón de Guatuso para la toma de decisiones.

f. Atlas del desarrollo local del cantón de Guatuso.

Esto permitió motivar a la población para que participe activamente en el proceso de formulación del plan. Por otra parte, se realizó una capacitación en planificación estratégica municipal, dirigido a funcionarios municipales de Guatuso, Los Chiles y Upala.

\section{Diagnóstico territorial}

La fase de diagnóstico territorial consistió en recolectar información de las diferentes organizaciones con el objetivo de comprender el modelo de desarrollo de la localidad. Se consultó información de un total de diecinueve organizaciones, entre las que se destacan:

- Municipalidad de Guatuso

- Ministerio de Agricultura y Ganadería (MAG)

- Caja Costarricense de Seguro Social (CCSS)

- Comisión Nacional de Emergencias (CNE)

- Ministerio de Salud

- Ministerio de Educación Pública (MEP)

- Instituto de Desarrollo Agrario (IDA)

- Instituto Geográfico Nacional (IGN)

- Instituto Nacional de Estadística y Censos (INEC)

- Ministerio de Ambiente, Energía y Telecomunicaciones (Minaet)

- Ministerio de Obras Públicas y Transportes (MOPT)

- Instituto Costarricense de Electricidad (ICE) 


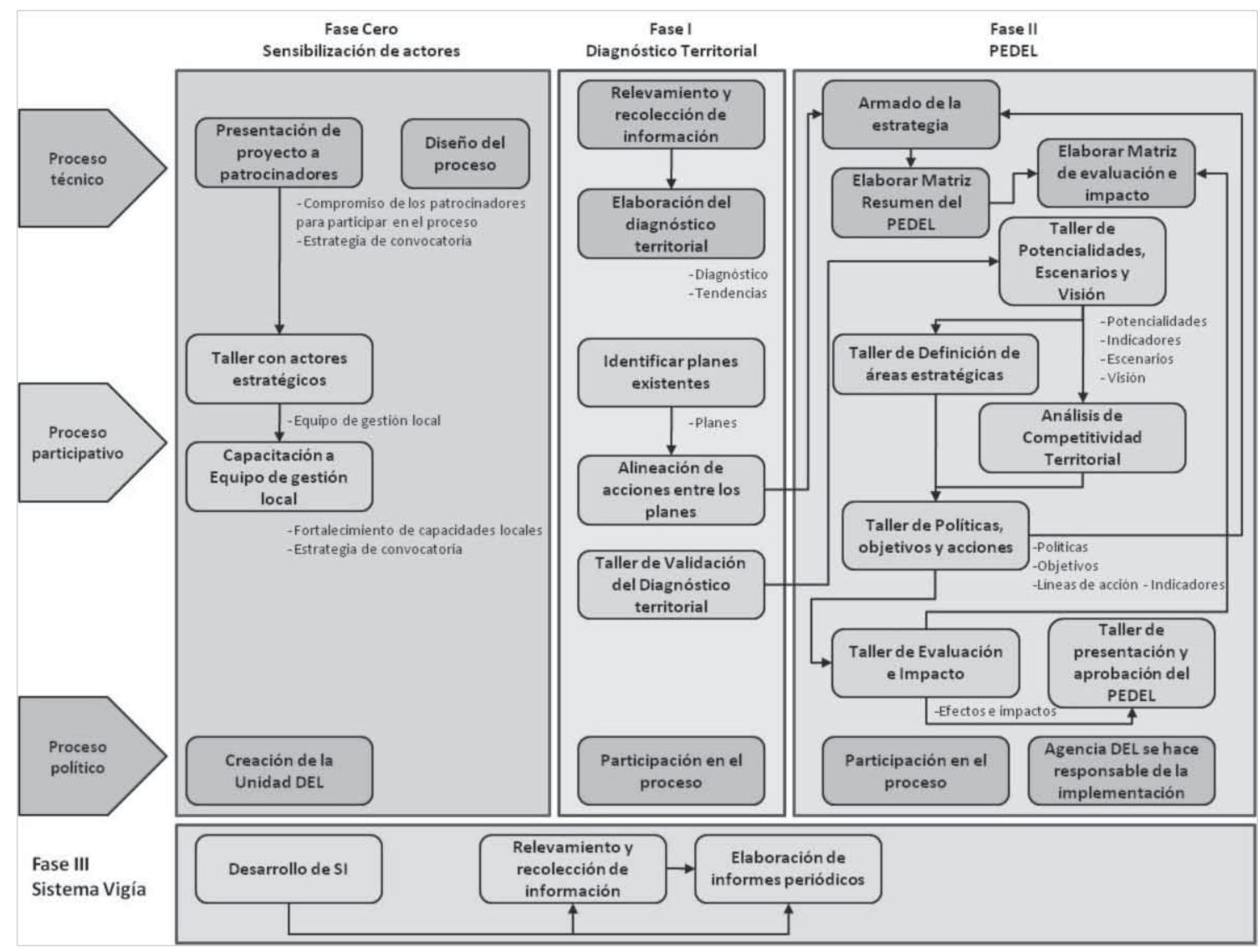

Figura I. Esquema metodológico propuesto para un plan estratégico territorial.

- Instituto Nacional de Biodiversidad (INBIO)

- Centro Científico Tropical

- Instituto Meteorológico Nacional (IMN)

- Programa de Naciones Unidas para el Desarrollo (PNUD)

- Ministerio de Planificación Nacional y Política Económica (Mideplan)

- Promotora del Comercio Exterior de Costa Rica (Procomer)

- Observatorio del Desarrollo de la Universidad de Costa Rica

Además, el SIR-ZEE se dio a la tarea de realizar una serie de trabajos de campo para desarrollar nuevas capas de información, las cuales son:

- Infraestructura comunal de los cantones de Guatuso y Los Chiles: esta capa se compone por salones comunales, iglesias, plazas de fútbol, cementerios, redondeles, puestos de fuerza pública y radio bases de telefonía celular.

- Puentes y pasos de alcantarilla de los cantones de Guatuso y Los Chiles: mediante la información contenida en esta capa, se ubicaron geográficamente los puentes y pasos de alcantarilla. También, se tomaron sus dimensiones y el estado de los mismos.

- Clasificación de la red vial por superficie de rodamiento.

- Actualización de la capa de Ebais y centros educativos de los cantones de Guatuso y Los Chiles.

- Levantamiento de las líneas de distribución de las Asadas de la cuenca del Río Frío, en conjunto con el Proyecto Río Frío.

Con la información de las organizaciones mencionadas anteriormente, se han elaborado dos revistas: Infraestructura comunal y estado de los puentes del cantón de Guatuso, y Atlas del 
desarrollo local del cantón de Guatuso. Además, se ha elaborado una serie de CD interactivos para que la población con dificultades de acceso a Internet pueda contar con la información. Asimismo, la información se encuentra publicada en la página del SIR-ZEE (www.sirzee.itcr.ac.cr).

\section{PEDEL}

En la actualidad, aún se está en la fase de formulación del Plan estratégico de desarrollo local. En conjunto con el Ministerio de Planificación (Mideplan), se abordó un primer taller para la identificación de prioridades cantonales con una participación activa de cien personas de la comunidad.

En dicha actividad se definió la visión cantonal de desarrollo:

Guatuso es un cantón líder en desarrollo sostenible, que brinda oportunidades reales y equitativas a sus habitantes, a fin de garantizar la calidad de vida, que promueve el acceso a la educación, la salud, el empleo y la protección del ambiente, y que cuenta con una población que participa activamente en los procesos de toma de decisiones.

También, se identificaron los objetivos cantonales de desarrollo y las líneas de acción prioritarias por área estratégica, así como los factores de éxito.

Las observaciones finales de la actividad fueron:

- Los participantes del taller de Guatuso están muy anuentes a intervenir activamente en los procesos de planificación del cantón y en acompañar la ejecución de las prioridades cantonales identificadas.

- Tienen muy altas expectativas con el nuevo gobierno local y esperanza en que la identificación de prioridades sirva para ordenar y promover el desarrollo integral del cantón. Por ello, es fundamental devolver la información a los participantes y que el gobierno local asuma que esta es únicamente una primera etapa y que continúe con el proceso de consulta comunal y distrital, con miras a la elaboración del Plan cantonal de desarrollo, con su respectivo Plan de acción, que incorpore mecanismos de seguimiento, evaluación y coordinación intercomunal e interinstitucional.

En el 2011 se identificó una lista de proyectos prioritarios para el cantón de Guatuso. Dichos proyectos fueron validados por un conjunto de actores de diferentes comunidades.

\section{Conclusiones y trabajos futuros}

En la Región Huetar Norte, la planificación es un tema incipiente y es necesario unir esfuerzos para que los gobiernos locales puedan contar con un plan estratégico a largo plazo, así como para que estos planes no queden guardados en las gavetas de los escritorios o sean desechados cuando se realicen elecciones municipales. Por otro lado, la población debe ser consciente de la importancia de su participación activa tanto en la formulación como en la ejecución del Plan, y debe procurar que se mejoren los procesos de rendición de cuentas.

Al considerar el párrafo anterior, vale la pena hacer hincapié en la frase de Sergio Boisier, que podría resumir la esencia de este análisis: "No hay ninguna receta que asegure el éxito en materia de desarrollo. Pero sí hay por lo menos dos afirmaciones ciertas: si el desarrollo se encuentra en nuestro futuro, no será con las ideas del pasado que lo alcanzaremos; si el desarrollo es un producto de la propia comunidad, no serán otros, sino sus propios miembros quienes lo construyan" (Boisier, 1998).

Se sabe que para poder planificar es necesario conocer y para ello es indispensable la información, la que luego se complementa con las percepciones que los actores manifiestan en los procesos participativos. Es por tal motivo que la información que está proporcionando el SIR-ZEE es de vital importancia para la toma de decisiones y, por lo tanto, para el proceso de planificación que se está llevando a cabo en este momento en el cantón de Guatuso. Sin embargo, aunque el SIR-ZEE ha conseguido vincularse con los gobiernos locales, todavía tiene como tarea lograr que la sociedad civil de la región utilice al cien por ciento las herramientas disponibles, y que estos se conviertan en productores de información para alimentar el sistema y mantenerlo actualizado. 


\section{Bibliografía}

Aguilar, Y.; Moya, N. \& Becerra, F. (2009). Aplicación del enfoque prospectivo para impulsar el Desarrollo Local en la comunidad 14 de Julio (Estudio de caso). Recuperado de http://www.eumed.net/libros/2009c/586/indice.htm

Boisier, S. (1998). Teorías y metáforas sobre desarrollo territorial. Revista austral de ciencias sociales. Obtenido el 7 de mayo del 2012, desde http://mingaonline. uach.cl/scielo.php?script=sci_arttext\&pid=S07 I 8| 795 | 99800010000 | \&lng=es\&nrm=iso

Buarque, S. (1999). Metodología de planejamento do desenvolvimento local e municipal sustentable. Recife: Instituto Interamericano de Cooperación para la Agricultura.

Cavuota, N. (2009). Herramientas para planificar el desarrollo: un Sistema de Información Territorial con enfoque de cadena de valor. Territorios, (20-2I). Obtenido desde http://redalyc.uaemex.mx/src/inicio/ ArtPdfRed.jsp?iCve=357| 4248009
Fundación Demuca. (2009). Guía de herramientas municipales para la promoción del desarrollo económico local. San José, Costa Rica.

Fundación Demuca \& CEDeT. (2009). Guía práctica para el Agente del Desarrollo Local de Centroamérica y República Domicana.

Real Academia Española. (200I). Diccionario de la lengua española (22. ${ }^{\text {a }}$ ed.). Consultado en http://www.rae.es/ rae.html

Ríos, D. (2007). Planeación prospectiva del desarrollo económico local. Obtenido desde http://dialnet.unirioja. es/servlet/articulo?codigo $=2668702$

Wild, J. \& López, O. (2008). Sistema de Información Regional de la Región Huetar Norte de Costa Rica: Sistematización de la experiencia. Cartago: Editorial Tecnológica de Costa Rica. 